\title{
Hyperthermia and Fibrinolytic Therapy Do Not Improve the Beneficial Effect of Radioimmunotherapy Following Cytoreductive Surgery in Rats with Peritoneal Carcinomatosis of Colorectal Origin
}

\author{
Frits Aarts, ${ }^{1}$ Thijs Hendriks, ${ }^{1}$ Otto C. Boerman, ${ }^{2}$ Wim J.G. Oyen, ${ }^{2}$ and Robert P. Bleichrodt ${ }^{1}$ \\ ${ }^{1}$ Department of Surgery, Radboud University Nijmegen Medical Centre, Nijmegen, The Netherlands \\ ${ }^{2}$ Department of Nuclear Medicine, Radboud University Nijmegen Medical Centre, Nijmegen, The \\ Netherlands
}

\begin{abstract}
Background and Objective: Cytoreductive surgery (CS) and heated intraperitoneal chemotherapy (HIPEC) are standard treatment for peritoneal carcinomatosis (PC) of colorectal cancer. Previously, we demonstrated that preclinical radioimmunotherapy (RIT) adjuvant to surgery in $P C$ is a good alternative for HIPEC. Now we aimed to improve the effectiveness of RIT by combining it with whole-body hyperthermia (WBH) or fibrinolytic therapy. Methods: Rats were inoculated intraperitoneally with colon carcinoma cells. Animals underwent CS, CS $+W B H\left(40^{\circ} \mathrm{C}, 3\right.$ hours $), C S+R I T\left(74 \mathrm{MBq}{ }^{177}\right.$ Lu-labeled MG1), or $C S+W B H+R I T$. In the second experiment, rats underwent $C S, C S+R I T, C S+$ recombinant tissue plasminogen activator (rtPA, twice daily, 3 days), or CS + RIT + rtPA. Results: Median survival after $C S$ and CS + WBH was 34 and 37 days. Median survival after CS + RIT or CS + RIT + WBH was 63 and 86 days $(p<0.0003, p<0.0006$ compared to $C S+W B H)$. Median survival after $C S$ and $C S+r t P A$ was 50 and 42 days $(p=0.1)$. Median survival was 106 days after $C S+$ RIT and 103 days after $C S+$ $R I T+r t P A(p<0.0001$ compared to $C S+r t P A)$. No difference was found between CS + RIT and CS + RIT $+r t P A(p=0.83)$. Conclusions: The application of WBH or rtPA in combination with adjuvant RIT after CS for the treatment of PC of colonic was feasible but did not significantly potentiate the efficacy of RIT.
\end{abstract}

Key words: peritoneal carcinomatosis, cytoreductive surgery, radioimmunotherapy, hyperthermia, fibrinolytic therapy, targeted radionuclide therapy

\section{INTRODUCTION}

Cytoreductive surgery (CS) followed by hyperthermic intraperitoneal chemotherapy (HIPEC) is considered one of the most promising treatments

Address reprint requests to: Fritz Aarts; Department of Surgery, Radboud University Nijmegen Medical Centre; P.O. Box 9101, 6500 HB Nijmegen, The Netherlands; Tel.: (+31) 24-3615333; Fax: (+31) 24-3540501

E-mail: F.Aarts@chir.umcn.nl of peritoneal carcinomatosis (PC) of colorectal carcinoma (CRC). ${ }^{1}$ In HIPEC, hyperthermia is used to enhance the chemosensitivity of the tumor. ${ }^{2} \mathrm{Me}-$ dian survival after these radical surgical debulking procedures (cytoreduction) is 13-34 months with a 5-year survival of $19 \%-27 \%$, which constitutes a significant improvement to palliative treatment consisting of chemotherapy and palliative surgery. ${ }^{3-7}$ However, the improved survival is reached at the cost of a considerable morbidity of $23 \%$ and 
a mortality of $4 \% .^{4,8}$ Thus, the search for alternative treatment strategies is warranted.

Radioimmunotherapy (RIT) using radiolabeled monoclonal antibodies directed against tumor-associated antigens may be an attractive anticancer therapy in patients with small-volume disease. ${ }^{9}$ In previous studies we have demonstrated the efficacy of RIT as an adjuvant to surgery in an animal model of PC. Survival in Wag/Rij rats with intraperitoneal CC-531 (colon carcinoma) tumors was significantly improved if CS was followed by adjuvant RIT using the ${ }^{177}$ lutetium-labeled anti-CC531 monoclonal antibody (MAb) MG1. ${ }^{10}$ The effect was most explicit when RIT treatment was administered immediately following surgery, as compared to 4 or 14 days after surgery. ${ }^{11}$ In rats with intraperitoneal CC531 tumors, RIT was found to be at least as effective as HIPEC in terms of survival after CS, and RIT induced fewer side effects than the adjuvant treatment with HIPEC. ${ }^{12}$ These experiments suggest that RIT is a promising therapeutic approach to ameliorate treatment of PC. The question now arises whether the efficacy of adjuvant RIT can be further improved.

It has been shown in preclinical studies with subcutaneously growing xenografts that the efficacy of RIT can be enhanced by locally applied hyperthermia. ${ }^{13}$ Hyperthermia increases tumor blood flow and interstitial $\mathrm{pO}_{2}$, reducing tumor hypoxia and disturbed cellular repair mechanisms. ${ }^{14-16}$

During surgical procedures, tissue damage causes deposition of fibrin, which may result in the formation of fibrinous adhesions. This phenomenon occurs as the result of a shift in the equilibrium between coagulation and fibrinolysis, in favor of the coagulation system. ${ }^{17-19}$ Tumor cells may be trapped in these fibrin clots and become inaccessible to therapeutic agents, such as radiolabeled antibodies, which may promote tumor growth. ${ }^{20-22}$ Postoperative fibrinolytic therapy might prevent these events from occurring. Here, we report on 2 separate animal studies where we investigated if either whole-body hyperthermia or fibrinolytic therapy (with tissue-type plasminogen activator [rtPA]) could improve the efficacy of adjuvant RIT, as applied after CS for PC of colonic origin.

\section{MATERIALS AND METHODS}

\section{Experimental Design}

Two (2) experiments were performed to study the effects of hyperthermia and rtPA, respec- tively. In both experiments, intraperitoneal tumor growth was induced by the inoculation of tumor cells. After 7 days, CS was performed. In the hyperthermia experiment, 60 rats, 15 in each treatment group, were randomly assigned to undergo either CS alone, CS followed by whole-body hyperthermia $(\mathrm{CS}+\mathrm{H})$, CS followed by RIT (CS + RIT), or CS followed by whole-body hyperthermia and RIT (CS + HRIT). In the fibrinolysis experiment, 58 rats were randomly assigned to undergo CS alone $(\mathrm{CS}, \mathrm{n}=15)$, CS followed by rtPA treatment $(\mathrm{CS}+\mathrm{rtPA}, \mathrm{n}=15)$, CS followed by RIT $(\mathrm{CS}+\mathrm{RIT}, \mathrm{n}=13)$, or a combination of both adjuvant treatments (CS + RIT + rtPA, $\mathrm{n}=$ $15)$. In both experiments, survival was determined and at autopsy the extent of tumor growth was scored.

\section{Cell Line}

The syngeneic rat colon carcinoma cell line CC531, originally induced in Wag/Rij rats by intravenous injection of 1,2-dimethylhydrazine, ${ }^{23}$ was cultured and maintained as monolayer in RPMI-1640 medium (Invitrogen, Breda, The Netherlands) supplemented with $10 \%$ fetal calf serum (GIBCO), $2 \mathrm{mM}$ L-glutamine, penicillin $(100 \mathrm{U} / \mathrm{mL})$, and streptomycin $(100 \mu \mathrm{g} / \mathrm{mL})$ at $37^{\circ} \mathrm{C}$ in a humidified atmosphere with $5 \% \mathrm{CO}_{2}$. Before inoculation, tumor cells were washed with $0.9 \%$ sodium chloride, disaggregated with $0.25 \%$ trypsin, and resuspended in $0.9 \%$ phosphatebuffered saline (PBS) to a concentration of $1 \times$ $10^{6}$ cells $/ \mathrm{mL} ; 2 \mathrm{~mL}$ of this cell suspension was injected intraperitoneally, as previously described. ${ }^{10}$

\begin{abstract}
Animals
Male Wag/Rij rats, 10 to 12 weeks old (body weight 240-285 g, Harlan, Horst, The Netherlands) were accustomed to laboratory conditions for at least 1 week before experimental use and housed under nonsterile standard conditions (temperature, $20^{\circ} \mathrm{C}-24^{\circ} \mathrm{C}$; relative humidity, 50\%-60\%; 12-hour light/dark cycle) in filtertopped cages ( 2 rats per cage), with free access to food (Ssniff, Bio Services, Uden, The Netherlands) and water. Physical condition was examined daily and body weight was recorded daily during the first 14 days after surgery by a biotechnician, who was blinded to the therapeutic regimen. Thereafter, body weight was recorded weekly. All experiments were approved by the
\end{abstract}


local Animal Welfare Committee of the Radboud University Nijmegen and were carried out in accordance with the Dutch Animal Welfare Act of 1997.

\section{Cytoreductive Surgery}

Surgical procedures were performed under general anesthesia using isoflurane $3 \%, \mathrm{O}_{2}$ and $\mathrm{N}_{2} \mathrm{O}$ 1:1. All rats underwent a midline laparotomy, followed by careful inspection of the abdominal contents for the presence of tumor. Tumor growth at each of the intra-abdominal quadrants was then scored as 0 (no macroscopic tumor growth), 1 (limited), 2 (moderate), or 3 (abundant). The peritoneal cancer index (PCI) was then the sum of the score for the four quadrants as described previously. ${ }^{10}$

In all treatment groups, CS was performed, including a routine omentectomy, and all macroscopic tumor deposits were removed surgically. Unresectable tumors were cauterized. After completion of the surgical cytoreduction, the abdominal wall was closed in 2 layers using continuous Vicryl 3-0 sutures for the muscular component and iron wound clips for the wound. After completion of the procedure, $10 \mathrm{~mL}$ of warmed, normal $\mathrm{NaCl}$ solution, $0.9 \%$ was given subcutaneously for rehydration. Thirty (30) minutes prior to surgery and once daily until the third postoperative day, rats were given buprenorphine $(5 \mu \mathrm{g}$, $0.1 \mathrm{~mL} / \mathrm{rat} / \mathrm{d}$ ) subcutaneously for analgesia.

\section{Radioimmunotherapy}

The murine MG1 MAb, an anti-CC531 IgG2a monoclonal antibody (Antibodies for Research Applications BV, Gouda, The Netherlands) that recognizes an $80-\mathrm{kDa}$ cell surface antigen on CC531 cells, was used in these studies. The MG1 MAb localizes preferentially in tumors when injected in rats bearing CC-531 tumors. ${ }^{24}$ Labeling of the antibody with ${ }^{177} \mathrm{Lu}$ (half-life 7 days, $\beta$ energy $149 \mathrm{keV}, \gamma$ - energy $208 \mathrm{keV}$, penetration depth $3 \mathrm{~mm}$ ) was carried out as previously described. ${ }^{10}$ In brief, the MAb was conjugated with 2-(4-isothiocyanatobenzyl)-diethylenetriaminepentaacetic acid (Macrocyclics, Dallas, TX), subsequently labeled with ${ }^{177}$ lutetium (IDB Holland, Baarle Nassau, The Netherlands), and purified by gel filtration on a PD10 column (Amersham, Pharmacia Biotech, Maarsen, The Netherlands). The purified ${ }^{177} \mathrm{Lu}-\mathrm{MG} 1$ preparation was diluted in PBS with $0.5 \%$ bovine serum albumin for injection. The specific activity of the administered
${ }^{177} \mathrm{Lu}-\mathrm{MG} 1$ preparation was $0.4 \mathrm{MBq} / \mu \mathrm{g}$. The ${ }^{177} \mathrm{Lu}$ labeling procedure was performed under strict metal-free conditions.

${ }^{177} \mathrm{Lu}$-labeled MG1 was used as the therapeutic agents in these experiments based on the favorable tumor-to-blood ratios obtained with ${ }^{111} \mathrm{In}$-labeled MG1 in this model as compared to ${ }^{125} \mathrm{I}-1 \mathrm{la}-$ beled MG1 $(9.2 \pm 5.3$ vs. $2.4 \pm 1.4$ with $p<$ 0.040 ) reported by Koppe et al. ${ }^{10}$ In addition, it was shown that intraperitoneal RIT using ${ }^{177} \mathrm{Lu}-$ labeled antibodies to treat small peritoneal metastases resulted in the highest percentage survival as compared to treatment with ${ }^{131} \mathrm{I}-,{ }^{186} \mathrm{Re}-$, or ${ }^{90}$ Y-labeled antibodies. ${ }^{25}$

RIT (185 $\mu \mathrm{g}$ MG1/rat, radiolabeled with 74 $\mathrm{MBq}{ }^{177} \mathrm{Lu}$ in $3.0 \mathrm{~mL}$ ) was intraperitoneally injected immediately following surgery, as this was determined to be the most optimal time for adjuvant administration in relation to $\mathrm{CS} .{ }^{11}$ In addition, this was presumed to be the most optimal time in relation to the application of hyperthermia. $^{26}$

\section{Hyperthermia}

Immediately upon recovery from general anesthesia, animals in the $\mathrm{CS}+\mathrm{H}$ group and the $\mathrm{CS}+\mathrm{HRIT}$ group were placed in an incubator preset at a temperature of $39^{\circ} \mathrm{C}$. After $30 \mathrm{~min}-$ utes, the temperature was raised to $40^{\circ} \mathrm{C}$. Inside the incubator, conditions were similar to those within the animal facility housing: a relative humidity between $20 \%$ and $40 \%$. General wholebody hyperthermia was maintained for 3 hours. The rectal temperature was monitored every 30 minutes.

\section{Fibrinolytic Therapy}

Immediately after the surgical procedure and after closure of the abdominal wall, the fibrinolytic therapy was administrated. Fibrinolytic therapy comprised of the intraperitoneal administration of $1.25 \mathrm{mg}$ human rtPA (Actilyse, Boehringer, Ingelheim, Germany) twice daily during the first 3 days after surgery. This dose was chosen because it was shown to be effective in reducing intraperitoneal abscess formation in a model of induced peritonitis in rats. ${ }^{27}$

\section{Follow-Up}

The primary endpoint in both experiments was survival. Animals were observed daily by an experienced biotechnician, blinded to the therapeu- 
tic regimen, who determined the humane endpoint. When this was reached (signs of massive hemorrhagic ascites, physical inactivity, or signs of intra-abdominal tumor growth with invalidating consequences), rats were killed by $\mathrm{O}_{2} / \mathrm{CO}_{2}$ asphyxiation and immediately dissected. At dissection, the intraperitoneal tumor growth was scored as described above. At 16 weeks postoperatively, the study was terminated and the remaining rats were killed and dissected. In case of absence of macroscopic tumor, all relevant organs, including the greater momentum, mesentery, and diaphragm, were removed for histology in order to examine tumor presence microscopically. Tissues were fixed in $1 \%$ formaldehyde, dehydrated, and embedded in paraffin. Sections were stained using hematoxylin and eosin and immunohistochemically stained using the murine MG1 antibody in combination with a peroxidaseconjugated horse-anti-mouse IgG antibody (Vector Laboratories Inc., Burlingame, CA, USA).

\section{Statistical Analysis}

Statistical analysis was performed using SPSS (Chicago, IL) software and Graphpad Prism version 4.0 (Graphpad Software Inc., San Diego, CA) for analysis. Comparison of dichotomous values was done using chi-square or Fisher exact test. Nonparametric testing was performed using 2-way analysis of variance testing. Survival curves were analyzed using Kaplan-Meier curves and compared by means of the log-rank test. Posttesting using Bonferroni was applied to correct for multiple groups. All tests were 2-sided; the level of statistical significance was set at a $p$ value of $<0.05$.

\section{RESULTS}

\section{Hyperthermia}

\section{Surgery and adjuvant treatment}

Preoperative clinical condition and body weight did not differ between groups. At laparotomy, tumor nodules were often present in the omentum, liver hilum, mesentery, and gonadal fat pads (1-3 mm diameter). Median PCI score at time of surgery was 8 (range $0-12$, Table 1) and did not differ between groups $(p=0.59)$. After CS, 2 of the 15 animals in the CS group had residual disease after surgery. During surgery, a bowel perforation occurred in 1 rat. No animals died during or immediately after surgery. Mean rectal temperatures during hyperthermia were equal in both hyperthermia groups: $39.4^{\circ} \mathrm{C} \pm 0.8^{\circ} \mathrm{C}$ in the $\mathrm{CS}+\mathrm{H}$ group and $39.5^{\circ} \pm$ $1.2^{\circ} \mathrm{C}$ in the $\mathrm{CS}+\mathrm{HRIT}$ group, respectively. Two (2) animals were euthanized directly after hyper-

\begin{tabular}{|c|c|c|c|c|c|c|c|c|}
\hline \multirow{2}{*}{$\begin{array}{l}\text { Disease } \\
\text { characteristics }\end{array}$} & \multicolumn{4}{|c|}{ Hyperthermia } & \multicolumn{4}{|c|}{ Fibrinolytic therapy } \\
\hline & $C S$ & $C S+H$ & $C S+R T$ & $C S+H R I T$ & $C S$ & $C S+r t P A$ & $C S+R I T$ & $C S+R I T+r t P A$ \\
\hline $\begin{array}{l}\text { Body weight } \\
\text { preoperative }\end{array}$ & $\begin{array}{c}264 \\
(246-281)\end{array}$ & $\begin{array}{c}265 \\
(251-278)\end{array}$ & $\begin{array}{c}265 \\
(249-282)\end{array}$ & $\begin{array}{c}268 \\
(252-285)\end{array}$ & $\begin{array}{c}274 \\
(257-290)\end{array}$ & $\begin{array}{c}274 \\
(260-288)\end{array}$ & $\begin{array}{c}275 \\
(253-292)\end{array}$ & $\begin{array}{c}274 \\
(263-287)\end{array}$ \\
\hline \multicolumn{9}{|l|}{ Tumor score per site } \\
\hline Greater omentum & $2(1-3)$ & $2(0-3)$ & $2(1-3)$ & $2(2-3)$ & $1(1)$ & $1(1-3)$ & $1(1)$ & $1(1)$ \\
\hline Liver hilum & $2(1-3)$ & $2(0-2)$ & $2(0-3)$ & $2(1-2)$ & $1(0-2)$ & $1(1)$ & $1(0-1)$ & $1(1-2)$ \\
\hline Perisplenic & $1(0-2)$ & $1(0-2)$ & $1(0-2)$ & $1(0-1)$ & $1(0-1)$ & $1(1-0)$ & $0(0-1)$ & $1(0-1)$ \\
\hline Mesentery & $1(0-2)$ & $1(0-2)$ & $1(0-2)$ & $2(1-3)$ & $1(0-1)$ & $1(0-3)$ & $1(0-1)$ & $1(0-1)$ \\
\hline Gonadal fat pads & $1(0-2)$ & $1(0-2)$ & $1(0-2)$ & $1(0-2)$ & $1(0-1)$ & $0(0-1)$ & $1(0-1)$ & $0(0-1)$ \\
\hline Diaphragm & $0(0-1)$ & $0(0-1)$ & 0 & 0 & $0(0)$ & $0(0)$ & $0(0)$ & $0(0)$ \\
\hline Parietal peritoneum & $1(0-2)$ & $1(0-2)$ & $1(0-2)$ & $1(1-2)$ & $1(0-1)$ & $0(0-1)$ & $1(0-1)$ & $0(0-3)$ \\
\hline Total & $8(4-11)$ & $8(0-12)$ & $9(3-10)$ & $9(7-11)$ & $5(4-6)$ & $5(4-10)$ & $5(3-8)$ & $5(4-6)$ \\
\hline \multicolumn{9}{|l|}{$\begin{array}{l}\text { Resection } \\
\text { macroscopically } \\
\text { Complete }\end{array}$} \\
\hline Yes & 13 & 15 & 15 & 15 & 15 & 15 & 13 & 15 \\
\hline No & 2 & 0 & 0 & 0 & 0 & 0 & 0 & 0 \\
\hline
\end{tabular}

CS, cytoreductive surgery; H, whole-body hypothermia; RIT, radioimmunotherapy; HRIT, whole-body hypothermia and radioimmunotherapy; rtPA, tissue-type plasminogen activator.

Results are median (range). 


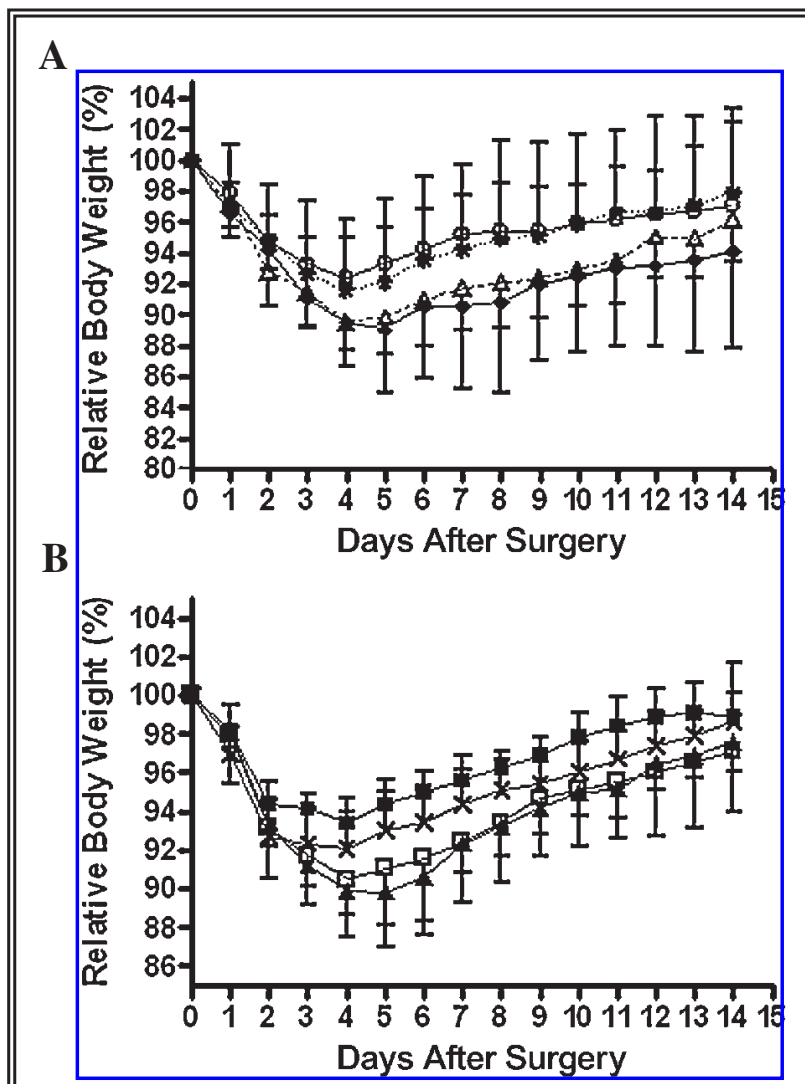

Figure 1. Course of body weight during the first 2 weeks after cytoreductive surgery. Body weight (mean + standard deviation) is given relative to weight at operation. (A) Effect of hyperthermia; (B) effect of tissue-type plasminogen activator (rtPA). $\bigcirc, \mathrm{CS} ; *, \mathrm{CS}+\mathrm{H} ; \triangle, \mathrm{CS}+\mathrm{RIT} ; \diamond, \mathrm{CS}+$ HRIT; $\square$, CS; $\boldsymbol{x}, \mathrm{CS}+\mathrm{rtPA} ; \boldsymbol{\Delta}, \mathrm{CS}+\mathrm{RIT} ; \square, \mathrm{CS}+$ RIT + rtPA. See text for description of groups.

thermia, 1 in the $\mathrm{CS}+\mathrm{H}$ group and 1 in the $\mathrm{CS}+$ HRIT group. Two (2) animals showed massive unexplained weight loss ( 1 each in the CS and CS + HRIT groups) and were euthanized 4 days after surgery. All animals lost weight after surgery (Fig. 1A). Maximum weight loss was $7.6 \% \pm 1.3 \%$ (mean and standard deviation) and $8.5 \% \pm 0.8 \%$ for the CS and CS + H groups and $10.3 \% \pm 0.4 \%$ and $10.7 \% \pm 0.7 \%$ for the CS + RIT and CS + HRIT groups, respectively. At day 4 and day 5 after surgery, weight loss was significantly higher in the CS + RIT group than in the CS group $(p<$ $0.05)$. In addition, from day 5 until day 9 , animals that were treated with CS + HRIT lost significantly more body weight as compared to animals treated with CS alone $(p<0.05)$.

\section{Follow-up}

Forty-nine (49) out of 56 animals reached the humane endpoint within 16 weeks after surgery. At time of death, mean PCI in the CS, CS + H, CS + RIT, and CS + HRIT groups was 20 (range 16-21), 20 (range 14-21), 17 (range 12-20), and 15 (range 2-21), respectively (Fig. 2A). The PCI was significantly lower in the CS + HRIT group than in both the CS $(p<0.01)$ and $\mathrm{CS}+\mathrm{H}(p<$ $0.05)$ groups. All these animals showed hemorrhagic ascites. At dissection, the volume of ascites was equal in all 4 groups (mean $19.9 \mathrm{~g}, p=0.35$ ). Median survival after CS was 34 days (range 25-112, end of study) and after CS + H, 37 days (range 15-83, Fig. 3A). The groups that were treated with adjuvant RIT survived significantly longer; after CS + RIT, median survival was 63 days (range $39-112, p<0.0003$ vs. $\mathrm{CS}+\mathrm{H}$ ) and after CS + HRIT 86 days (range 35-112,

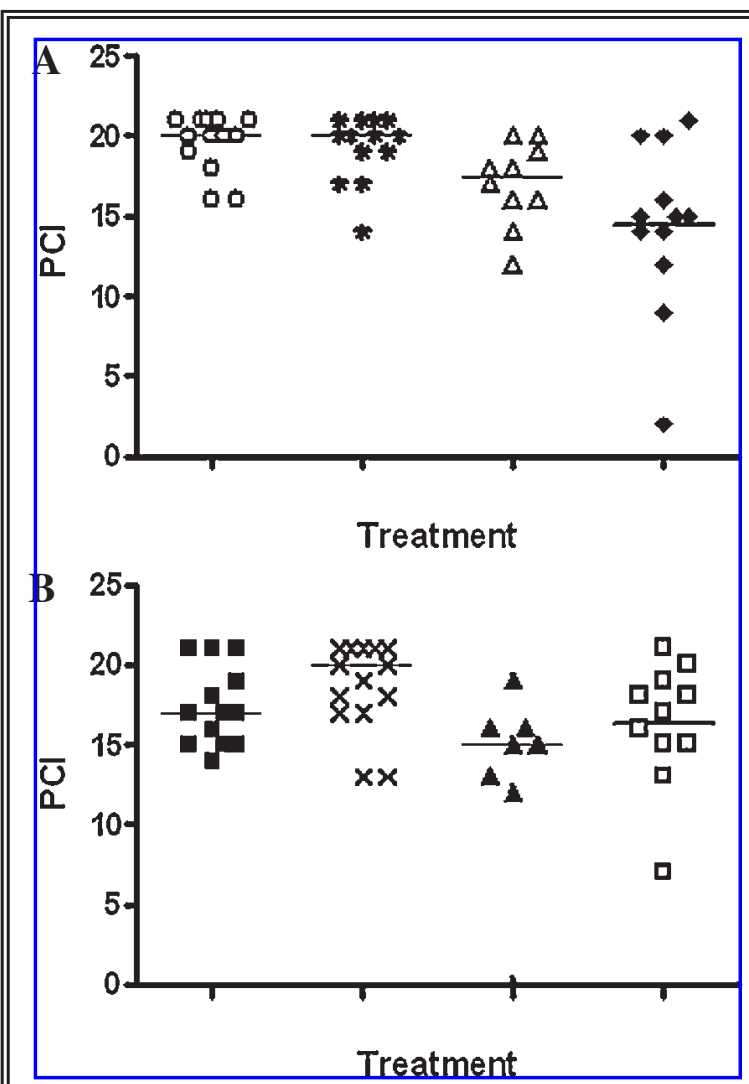

Figure 2. Peritoneal cancer index (PCI) at time of death. Individual values are given together with the median (horizontal bar). In the hyperthermia experiment (A), the PCI was significantly lower in the CS + HRIT group than in both the $\mathrm{CS}(p<0.01)$ and $\mathrm{CS}+\mathrm{H}(p<0.05)$ groups. In the rtPA experiment (B), the PCI was significantly lower in the CS + RIT group than in both the CS $(p<0.01)$ and CS + RIT + rtPA ( $p<0.05$ and $p<0.01$, respectively) groups. $\bigcirc$, CS; *, CS + H; $\triangle$, CS + RIT;, CS + HRIT;, CS; $*$, CS + rtPA; $\boldsymbol{\Delta}$, CS + RIT; $\square$, CS + RIT + rtPA. See text for description of groups. 


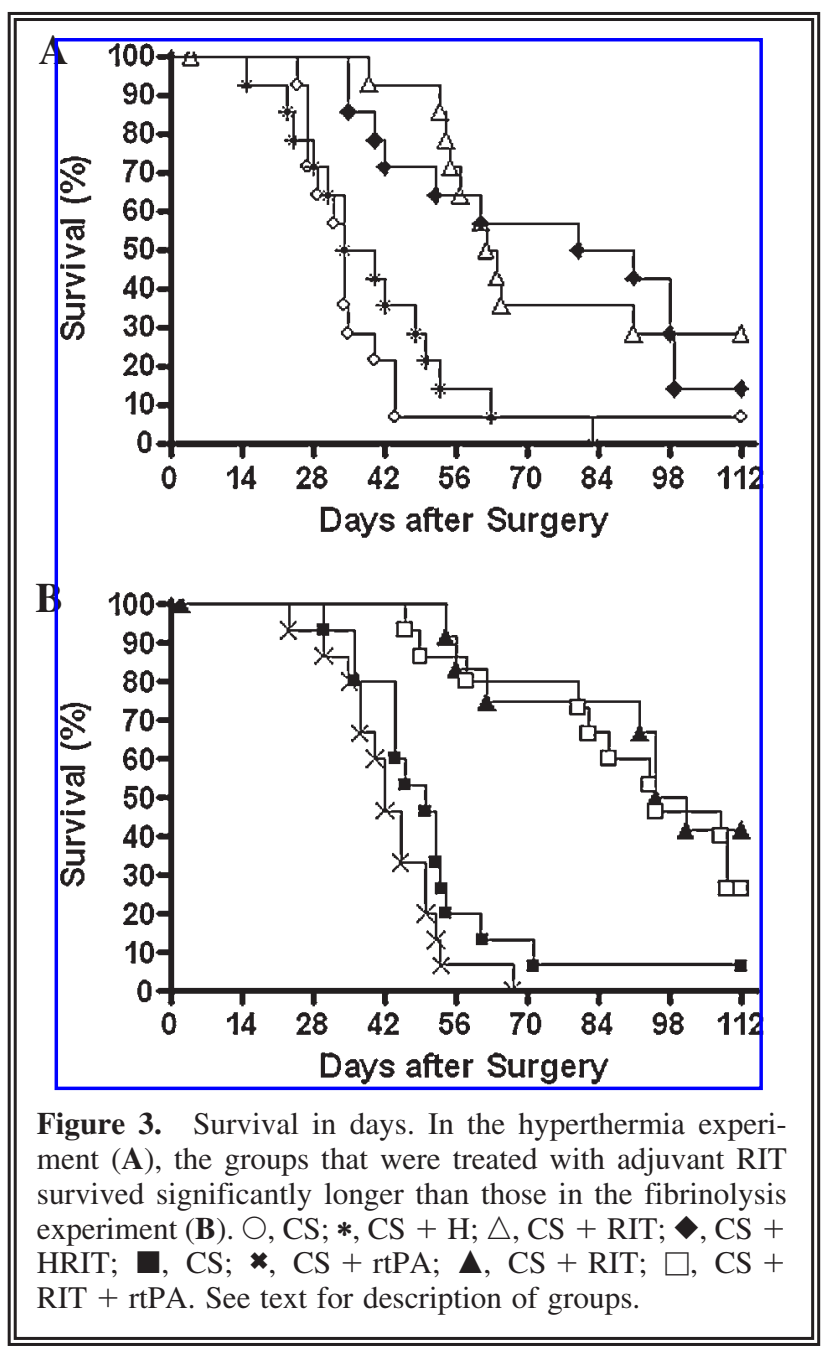

$p<0.0006$ vs. $\mathrm{CS}+\mathrm{H})$. However, the apparent difference between the latter groups remained nonsignificant $(p=0.72)$.

Seven (7) rats (1 in the CS group, 4 in the CS + RIT group, and 2 in the CS + HRIT group) survived until 16 weeks after surgery and were killed subsequently. Three (3) of these animals had macroscopic tumor ( 2 in the CS + RIT group and 1 in the CS + HRIT group). Microscopic investigation of the remaining 4 animals did not reveal any tumor.

\section{Fibrinolytic Therapy}

\section{Surgery and adjuvant treatment}

Preoperative clinical condition and body weight did not differ between groups. At laparotomy, tumor nodules were present in the omentum, liver hilum, mesentery, and gonadal fat pads (1-3 mm diameter). The median PCI score at time of surgery was 5 (range 3-10) and was similar in all groups ( $p=0.28$, Table 1$)$. No animals died during or immediately after surgery. One (1) animal in the CS + RIT group was killed 2 days after surgery due to a burst abdomen. All animals lost weight after surgery (Fig. 1B). Maximum weight loss was $6.6 \% \pm 2.6 \%$ and $7.9 \% \pm 3.8 \%$ in the $\mathrm{CS}$ and $\mathrm{CS}+\mathrm{rtPA}$ groups and $10.1 \% \pm 2.6 \%$ and $9.5 \% \pm 5.8 \%$ in the CS + RIT and CS + RIT + rtPA groups, respectively. There was a significant difference in weight loss between the CS and $\mathrm{CS}+\mathrm{rtPA}$ groups from day 2 until day $4(p<$ $0.05)$. In addition, from day 3 up until day 13, animals treated with RIT or RIT + rtPA lost significantly more weight than animals treated with CS alone ( $p<0.01$ and $p<0.05$, respectively).

\section{Follow-up}

Forty-seven (47) out of 57 animals reached the humane endpoint before the termination of the experiment 16 weeks after surgery. The mean PCI at the time of death in the CS, CS + rtPA, CS + RIT, and CS + RIT + rtPA groups was 17 (range 14-21), 20 (range 13-21), 15 (range 0-19), and 17 (range 7-21), respectively (Fig. 2B). The PCI was significantly lower in the CS + RIT group than in both the CS alone $(p<0.01)$ and $\mathrm{CS}+$ $\mathrm{RIT}+\mathrm{rtPA} \quad(p<0.05$ and $p<0.01$, respectively) groups. No difference in the volume of ascites was found between the groups at the time of sacrifice. Median survival after CS alone and $\mathrm{CS}+\mathrm{rtPA}$ was 50 days (range 30-112) and 42 days (range 23-67) $(p=0.09)$, respectively. The groups that were treated with adjuvant RIT survived significantly longer (Fig. 3B). The median survival of animals that were treated with $\mathrm{CS}+$ RIT was 106 days (range 54-112), and those treated with CS + RIT + rtPA survived for 103 days (range 46-112; $p<0.0001$ when compared to CS + rtPA for both these treatment groups). There was, however, no difference in survival between both RIT-treated groups $(p=0.52)$.

Of the 10 animals that were still alive at the end of the experiment ( 1 in the CS group, 5 in the CS + RIT group, and 4 in the CS + RIT + rtPA group), 3 animals ( 2 in the CS + RIT group and 1 in the CS + RIT + rtPA group) showed macroscopic and 1 (in the CS + RIT group) showed microscopic tumor at dissection.

\section{DISCUSSION}

The present data demonstrate that application of RIT immediately after surgery more than doubles 
survival time. However, the results of both experiments demonstrate that the efficacy of RIT as an adjuvant treatment after CS for PC is not significantly improved by hyperthermic treatment or the use of fibrinolytic drugs in an established model of PC in the rat.

In Wag/Rij rats, the intraperitoneally growing syngeneic rat colon carcinoma cell line CC531 has been shown to present a highly reproducible model of PC that is similar to the human entity of PC. ${ }^{28}$ We have shown before that RIT with ${ }^{177} \mathrm{Lu}$-labeled MG1, administered after CS, is a promising adjuvant treatment. ${ }^{10,11}$

In the clinical situation, hyperthermia may enhance the effect of radiotherapy during 1 hour and combined with a single high dose of radiotherapy. ${ }^{29}$ The application of prolonged (3-6 hours) mild whole-body hyperthermia $\left(40^{\circ} \mathrm{C}\right)$ in combination with low-dose RIT $\left({ }^{131}\right.$ I-labeled anti-carcinoembryonic antigen (CEA) antibody F-33-104) delayed the growth of subcutaneously growing human colon cancer xenografts. ${ }^{30}$ The 6-hour course of hyperthermia led to a mortality of $23 \%$. Probably, a shorter time of hyperthermia is effective as well. It has been shown that the effects of hyperthermia (i.e., increased blood flow and oxygenation), even when applied for $60 \mathrm{~min}$ utes only, lasted for 24 hours. ${ }^{31}$ Kinuya et al. ${ }^{32}$ demonstrated an increased (2.4-fold) accumulation of the radiolabeled antibody in subcutaneously growing human colon cancer xenografts in nude mice, resulting in improved therapeutic efficacy when hyperthermia was applied directly after antibody injection. If hyperthermia was applied either 2 days prior to or 2 days after the injection of the radiolabeled MAb, the opposite effect was observed. In the present study, we therefore applied whole-body hyperthermia of $40^{\circ} \mathrm{C}$ immediately after the intraperitoneal injection of the radiolabeled antibody. This procedure resulted in a hyperthermia-related mortality of $7 \%$. In contrast to the above-mentioned studies, the present study failed to show a significantly enhanced therapeutic efficacy of RIT after hyperthermia, although the median survival increased by $36 \%$. The lack of a significant effect of the adjuvant application of mild hyperthermia in our model may be due to various reasons. The microenvironmental factors influenced by hyperthermia are (1) perfusion, (2) vascular permeability, (3) $\mathrm{pO}_{2}$, (4) $\mathrm{pH}$, and (5) interstitial fluid pressure in the tumor. ${ }^{31}$

An increase in tumor perfusion reduces hypoxia, which may enhance radiosensitivity. This has been reported by Mittal and colleagues, ${ }^{33}$ who used an ${ }^{131}$ I-labeled anti-CEA antibody in combination with temperatures between $41^{\circ} \mathrm{C}$ and $43^{\circ} \mathrm{C}$ for the duration of 45 minutes to treat human colon cancer xenografts. The authors concluded that hyperthermia increased the effectiveness of RIT but without an increase uptake of the antibody in the tumor. Most likely, intraperitoneally growing small tumor nodules $(<1 \mathrm{~mm})$ originating from CC531 cells only have a small hypoxic fraction. This may explain the lack of increased efficacy in our model.

Increased vascular permeability is reported to occur at temperatures exceeding $41.5^{\circ} \mathrm{C}$. Under the present experimental conditions, these high temperatures would lead to an unacceptably high morbidity and thus is not applicable in this model.

Abdominal surgery inevitably results in peritoneal trauma followed by the deposition of fibrin. ${ }^{34}$ Tumor cells can be encapsulated within this fibrin network and as such would become less accessible to intraperitoneal RIT. ${ }^{35}$ Administration of rtPA will lyse fibrin and may counteract this effect. In the present study, the administration of rtPA did not result in increased survival. This may be due to ineffective fibrinolysis. However, using a similar dose, Buyne et al. reported that 2 intraperitoneal injections with $1.25 \mathrm{mg}$ rtPA reduced abscess formation with $30 \%$ in an intra-abdominal sepsis model. ${ }^{27}$ Jacquet and colleagues found that intraoperative administration of rtPA $(5 \mathrm{mg} / \mathrm{kg})$ resulted in a decreased number of tumor implants when colon carcinoma cells were administered intraperitoneally after creating peritoneal wounds. ${ }^{36}$ The applied dose of $1.25 \mathrm{mg}$ twice daily for 3 days, however, did not result in an improved survival in the present study. Apparently, in the presently used model, fibrin deposition is not a factor limiting the effectiveness of RIT. The use of an alpha-emitter in this model may have been more appropriate than the use of Lu-177. One might argue that the penetration range of the alpha particles $(<70 \mu \mathrm{m})$ is more suited for treating tumor cell clusters and very small tumor nodules than the penetration range of the beta particles emitted by Lu-177 $(<3.3 \mathrm{~mm}){ }^{37}$

In summary, we showed that the administration of RIT adjuvant to CS in a model of induced PC of colonic origin improves survival. In addition, we demonstrated that the application of mild WBH or rtPA as adjunct to RIT and CS is feasible but did not show a significant additive effect to the combined treatment of RIT and CS in our model of PC of CRC. 


\section{CONCLUSIONS}

This study showed that the application of combined WBH and RIT adjuvant to CS is feasible but did not significantly improve the efficacy of the combined treatment of RIT and CS in a model of PC of CRC. The combination of both treatment modalities, however, could be an effective adjuvant treatment for PC of CRC in cases of hypoxic tumors. In addition, the use of fibrinolytic therapy in the model of induced PC may be further explored using alpha-emitters as radionuclides.

\section{ACKNOWLEDGMENTS}

The authors wish to thank Ben de Man and Roger Lomme (Radboud University Nijmegen Medical Center, Surgical Research Laboratory) for their excellent assistance during the operative procedures and Henny Eikholt (Radboud University Nijmegen Medical Centre, Central Animal Laboratory) for his assistance in the animal experiments.

\section{REFERENCES}

1. Koppe MJ, Boerman OC, Oyen WJ, et al. Peritoneal carcinomatosis of colorectal origin: incidence and current treatment strategies. Ann Surg 2006;243:212.

2. van Ruth S, Verwaal VJ, Hart AA, et al. Heat penetration in locally applied hyperthermia in the abdomen during intra-operative hyperthermic intraperitoneal chemotherapy. Anticancer Res 2003;23:1501.

3. Sugarbaker PH. Colorectal carcinomatosis: a new oncologic frontier. Curr Opin Oncol 2005; 17:397.

4. Glehen O, Kwiatkowski F, Sugarbaker PH, et al. Cytoreductive surgery combined with perioperative intraperitoneal chemotherapy for the management of peritoneal carcinomatosis from colorectal cancer: a multi-institutional study. J Clin Oncol 2004;22:3284.

5. Verwaal VJ, van RS, de BE, et al. Randomized trial of cytoreduction and hyperthermic intraperitoneal chemotherapy versus systemic chemotherapy and palliative surgery in patients with peritoneal carcinomatosis of colorectal cancer. J Clin Oncol 2003;21:3737.

6. Glehen O, Cotte E, Schreiber V, et al. Intraperitoneal chemohyperthermia and attempted cytoreductive surgery in patients with peritoneal carcinomatosis of colorectal origin. Br J Surg 2004;91:747.

7. Culliford AT, Brooks AD, Sharma S, et al. Surgical debulking and intraperitoneal chemotherapy for established peritoneal metastases from colon and appendix cancer. Ann Surg Oncol 2001;8:787.
8. Elias D, Blot F, El OA, et al. Curative treatment of peritoneal carcinomatosis arising from colorectal cancer by complete resection and intraperitoneal chemotherapy. Cancer 2001;92:71.

9. Koppe MJ, Bleichrodt RP, Oyen WJ, et al. Radioimmunotherapy and colorectal cancer. $\underline{B r} \quad J \quad$ Surg 2005;92:264.

10. Koppe MJ, Hendriks T, Boerman OC, et al. Radioimmunotherapy is an effective adjuvant treatment modality after cytoreductive surgery of peritoneal carcinomatosis of colonic origin. J Nucl Med 2006;47:1867.

11. Aarts F, Koppe MJ, Hendriks T, et al. Timing of adjuvant radioimmunotherapy after cytoreductive surgery in experimental peritoneal carcinomatosis of colorectal origin. Ann Surg Oncol 2007;14:533.

12. Aarts F, Hendriks T, Boerman OC, et al. A comparison between radioimmunotherapy and hyperthermic intraperitoneal chemotherapy for the treatment of peritoneal carcinomatosis of colonic origin in rats. Ann Surg Oncol 2007; 14:3274.

13. Kinuya S, Yokoyama K, Hiramatsu T, et al. Optimal timing of administration of hyperthermia in combined radioimmunotherapy. Cancer Biother Radiopharm 2000;15:373.

14. Horsman MR. Tissue physiology and the response to heat. Int J Hyperthermia 2006;22:197.

15. Gerner EW, Connor WG, Boone ML, et al. The potential of localized heating as a adjunct to radiation therapy. Radiology 1975;116:433.

16. Schlemmer M, Lindner LH, bdel-Rahman S, et al. [Principles, technology and indication of hyperthermia and part body hyperthermia]. Radiologe 2004;44:301.

17. Holmdahl L, Eriksson E, Eriksson BI, et al. Depression of peritoneal fibrinolysis during operation is a local response to trauma. Surgery 1998;123:539.

18. Scott-Coombes D, Whawell S, Vipond MN, et al. Human intraperitoneal fibrinolytic response to elective surgery. Br J Surg 1995;82:414.

19. Neudecker J, Junghans T, Raue W, et al. Fibrinolytic capacity in peritoneal fluid after laparoscopic and conventional colorectal resection: data from a randomized controlled trial. Langenbecks Arch Surg 2005;390:523.

20. Nagy JA, Meyers MS, Masse EM, et al. Pathogenesis of ascites tumor growth: fibrinogen influx and fibrin accumulation in tissues lining the peritoneal cavity. $\underline{\mathrm{Can}}$ cer Res 1995;55:369.

21. Biggerstaff JP, Seth N, Amirkhosravi A, et al. Soluble fibrin augments platelet/tumor cell adherence in vitro and in vivo, and enhances experimental metastasis. $\underline{\text { Clin }}$ Exp Metastasis 1999;17:723.

22. Dvorak HF. Rous-Whipple Award Lecture. How tumors make bad blood vessels and stroma. Am J Pathol 2003;162:1747.

23. Zedeck MS. A model system for studies of colon carcinogenesis: tumor induction by a single injection of methylazoxymethanol acetate. J Natl Cancer Inst 1974;53:1419.

24. Hagenaars M, Koelemij R, Ensink NG, et al. The development of novel mouse monoclonal antibodies 
against the CC531 rat colon adenocarcinoma. Clin Exp Metastasis 2000;18:281.

25. Koppe MJ, Bleichrodt RP, Soede AC, et al. Biodistribution and therapeutic efficacy of (125/131)I-, (186)Re, (88/90)Y-, or (177)Lu-labeled monoclonal antibody $\mathrm{MN}-14$ to carcinoembryonic antigen in mice with small peritoneal metastases of colorectal origin. $\underline{\mathrm{J} \mathrm{Nucl} \mathrm{Med}}$ 2004;45:1224.

26. Kinuya S, Yokoyama K, Hiramatsu T, et al. Optimal timing of administration of hyperthermia in combined radioimmunotherapy. Cancer Biother Radiopharm 2000; $15: 373$.

27. Buyne OR, Bleichrodt RP, van GH, et al. Tissue-type plasminogen activator prevents formation of intra-abdominal abscesses after surgical treatment of secondary peritonitis in a rat model. Int J Colorectal Dis 2007;22:819.

28. Lopes Cardozo AM, Gupta A, Koppe MJ, et al. Metastatic pattern of CC531 colon carcinoma cells in the abdominal cavity: an experimental model of peritoneal carcinomatosis in rats. Eur J Surg Oncol 2001;27:359.

29. Wust P, Hildebrandt B, Sreenivasa G, et al. Hyperthermia in combined treatment of cancer. Lancet Oncol 2002;3:487.

30. Saga T, Sakahara H, Nakamoto Y, et al. Enhancement of the therapeutic outcome of radio-immunotherapy by combination with whole-body mild hyperthermia. Eur J Cancer 2001;37:1429.
31. Song CW, Park HJ, Lee CK, et al. Implications of increased tumor blood flow and oxygenation caused by mild temperature hyperthermia in tumor treatment. Int J Hyperthermia 2005;21:761.

32. Kinuya S, Yokoyama K, Hiramatsu T, et al. Optimal timing of administration of hyperthermia in combined radioimmunotherapy. Cancer Biother Radiopharm 2000;15:373.

33. Mittal BB, Zimmer AM, Sathiaseelan V, et al. Effects of hyperthermia and iodine-131-labeled anticarcinoembryonic antigen monoclonal antibody on human tumor xenografts in nude mice. Cancer 1992; 70:2785.

34. Reijnen MM, Bleichrodt RP, Van Goor H. Pathophysiology of intra-abdominal adhesion and abscess formation, and the effect of hyaluronan. Br J Surg 2003; 90:533.

35. Oosterling SJ, van der Bij GJ, van EM, et al. Surgical trauma and peritoneal recurrence of colorectal carcinoma. Eur J Surg Oncol 2005;31:29.

36. Jacquet P, Stuart OA, Dalton R, et al. Effect of intraperitoneal chemotherapy and fibrinolytic therapy on tumor implantation in wound sites. J Surg Oncol 1996; 62:128.

37. Zalutsky MR, Reardon DA, Pozzi OR, et al. Targeted alpha-particle radiotherapy with (211)At-labeled monoclonal antibodies. Nucl Med Biol 2007;34:779. 


\section{This article has been cited by:}

1. Changlin Zhao, Congqi Dai, Xiaoyin Chen. 2012. Whole-body hyperthermia combined with hyperthermic intraperitoneal chemotherapy for the treatment of stage IV advanced gastric cancer. International Journal of Hyperthermia 1-7. [CrossRef]

2. Sophie E. Eriksson, Tomas Ohlsson , Rune Nilsson, Jan Tennvall . 2012. Treatment with Unlabeled mAb BR96 After Radioimmunotherapy with 177Lu-DOTA-BR96 in a Syngeneic Rat Colon Carcinoma Model. Cancer Biotherapy \& Radiopharmaceuticals 27:3, 175-182. [Abstract] [Full Text HTML] [Full Text PDF] [Full Text PDF with Links]

3. Sophie E. Eriksson, Tomas Ohlsson, Rune Nilsson, Jan Tennvall . 2012. Repeated Radioimmunotherapy with 177Lu-DOTABR96 in a Syngeneic Rat Colon Carcinoma Model. Cancer Biotherapy \& Radiopharmaceuticals 27:2, 134-140. [Abstract] [Full Text HTML] [Full Text PDF] [Full Text PDF with Links] 